\title{
Phytochemical Composition and Antibacterial Activities of the Ethyl Acetate Leaf Extract of Ocimum basilicum
}

Japheth Wambani Rapando ( $\square$ wambanijr@gmail.com )

Kenyatta University https://orcid.org/0000-0001-7420-7029

Mathew Piero Ngugi

Kenyatta University

Margaret Muturi

Kenyatta University

James Opiyo Ogutu

Kenyatta University

Research article

Keywords: Antibacterial, Ocimum basilicum, extract, phytochemicals

Posted Date: September 28th, 2020

DOI: https://doi.org/10.21203/rs.3.rs-80006/v1

License: (c) (i) This work is licensed under a Creative Commons Attribution 4.0 International License.

Read Full License 


\section{Abstract}

\section{Introduction}

Antibiotic resistance is a serious threat to humankind. There is need for the development of new therapeutic options. Ocimum basilicum has been used traditionally as a medicinal herb against infectious diseases and as a food additive.

\section{Objective}

This study aimed at evaluating the phytochemical composition and in vitro antibacterial activities of the ethyl acetate leaf extract of Ocimum basilicum.

\section{Methods}

Ocimum basilicum leaves were collected from Mbeere, Embu County, Kenya and ethyl acetate extraction done at Kenyatta University. Phytochemical composition was evaluated by Gas chromatograph mass spectrophotometry while antibacterial activities were evaluated by disc diffusion and broth microdilution methods. The test microorganisms were methicillin resistant $S$. aureus, $S$. aureus, P. aeruginosa and $E$. coli.

\section{Results}

The extract exhibited a broad spectrum antibacterial activity. The gram negative bacteria showed more susceptibility to the organic extract compared to the gram positive bacteria. Pseudomonas aeruginosa had the highest zone of inhibition $(27.00 \pm 2.00 \mathrm{~mm})$, while the $S$. aureus isolate had the lowest zone of inhibition (24.00 $\pm 1.00 \mathrm{~mm})$. Escherichia coli, S. aureus and MRSA species had an MIC of $62.5 \mathrm{mg} / \mathrm{ml}$ compared to $P$. aeruginosa with an MIC of $125 \mathrm{mg} / \mathrm{ml}$ when exposed to the leaf extract. The antibacterial activity could be attributed to the synergistic effects of phytochemicals such as terpenoids, alkaloids, essential oils, fatty acids, flavonoids and aldehydes that have been known to have antimicrobial properties. A total of 30 phytochemical compounds were eluted from the extract of $O$. basilicum. Nootkatone, a sesquiterpenoid had the highest concentration at $20.86 \mathrm{mg} / \mathrm{g}$. The compound 1,3-Dimethyl5 -isobutylcyclohexane, a fatty acid had the lowest concentration at $0.10 \mathrm{mg} / \mathrm{g}$.

\section{Conclusion}

O. basilicum has antibacterial activities on the tested pathogens and can be used to treat infections. Phytochemicals with antibacterial effects in the herb can be used as lead molecules in developing new antibacterial drugs.

\section{Introduction}


Infectious diseases account for $41 \%$, measured in Disability-Adjusted Life Years, of the global disease burden. That is far more than injuries (16\%) and close to non-infectious diseases (43\%) [1]. Among the major causes of this burden is the emergence of antibiotic resistance, a problem that is evolving towards being a global pandemic. It has resulted in the appearance of multidrug resistant bacteria, or superbugs $[2,3]$. Antibiotic resistance is a serious public health concern, especially in the developing countries with a high burden of infectious diseases [3]. Pathogens with a significant health threat include Methicilin resistant Staphylococcus aureus (MRSA), Staphylococcus aureus (S. aureus), Pseudomonas aeruginosa (P. aeruginosa) and Escherichia coli (E. coli). These species are responsible for a number of diseases including pulmonary infections, urinary tract infections, skin and soft tissue infections, cellulitis, folliculitis and septicaemia [4-8]. The problem posed by these antibiotic resistant pathogens has brought to the fore the need for the development of novel antibacterial agents [3].

Biologically active compounds that could be isolated and harnessed for their antibacterial, antiviral and antifungal activities are found, naturally, in plants. These active compounds include alkaloids, tannins, terpenoids, fatty acids and flavonoids. They are secondary metabolites with complex structures $[9,10]$. Their antimicrobial mechanisms include cell wall damage, cytoplasmic membrane damage, formation of reactive oxygen species, Deoxyribonucleic acid fragmentation, phosphatidylserine externalization, metacaspase activation, mitochondrial membrane depolarization, nuclear condensation, modulation of transcription factors, redox signaling and redox-sensitive transcription factors [11]. Exploitation of these factors can help in the development of better antimicrobial approaches. It is, therefore, conceivable that knowledge and data regarding the therapeutic potential of medicinal herbs is of great scientific interest as effective alternatives to the battle of antibiotic resistant microorganisms. Studies into the pharmacological activities of medicinal herbs and plants aim at providing empiric evidence on the use of traditional medicine, their pharmaceutical applications and commercialization of their active components.

Species of the genus Ocimum have been studied for their medicinal properties and some have been found to possess anti-inflammatory, analgesic, antidiabeteic, antioxidant and antimicrobial activities [12, $13,14]$. Ocimum basilicum is a herb that grows in the sub Saharan Africa, Asia pacific among other regions. Its leaves have been used among the Mbeere people of Kenya to treat infectious diseases [15]. Based on its use in Kenya, it was compelling to determine the antibacterial potential of its leaves on different bacterial species. The phytochemical compositions of this herb was also determined and associated with its antibacterial actions.

\section{Methodology}

\section{Plant Material}

Ocimum basilicum was obtained from Siakago, Kenya, on longitude $29^{\circ}$ and between latitudes $0^{\circ} 35^{\prime} 38^{\prime \prime} \mathrm{S}$ and $37^{0} 38^{\prime} 12^{\prime \prime} \mathrm{E}$ in January, 2017. Collection was based on its use locally. The plant was taxonomically identified and a voucher specimen deposited at the National Museum herbarium. The leaf samples were 
cleaned under running tap water and dried at room temperature to preserve heat sensitive molecules. Dried leaves were then ground to powder using an electric mill.

\section{Extract Preparation}

Five hundred grams of the powdered $O$. basilicum leaves were soaked in 1.5 liters of ethyl acetate (LOBA Chemie, Mumbai, India) in a volumetric flask for 72 hrs. The solution was sieved using a muslin cloth and filtered using a Rocker 400 vacuum pump into a conical flask. Concentration to remove the solvent was done by a rotating evaporator. Percentage yield was calculated on a dry weight basis. The extract was stored at $4^{\circ} \mathrm{C}$. This procedure adopted that used by Sui et al. with minor modifications (16).

$$
\text { Percentage Yield }=\frac{\text { Weight of Final Extract }}{\text { Weight of the Soaked Sample }} \times 100
$$

\section{Quantitative Phytochemical Analysis}

Quantitative phytochemical analysis was done according to the procedure by Arora and Saini [17]. One gram of the extract was weighed into a $1.5 \mathrm{ml}$ Eppendorf tube and dissolved using $1 \mathrm{ml}$ of ethyl acetate. The mixture was vortexed for 30 seconds and sonicated for five minutes using a Branson 2510E-DTE sonicator. The sample was spun in a centrifuge for 5 minutes at $1300 \mathrm{rpm}$. The supernatant was transferred into $2 \mathrm{ml}$ auto sampler vials and analyzed using a Gas Chromatograph Mass Spectrometry (7683 Agilent Technologies, Inc., Beijing, China).

\section{Antibacterial Assays}

Four concentrations of the extract $(1 \mathrm{~g} / \mathrm{ml}, 0.75 \mathrm{~g} / \mathrm{ml}, 0.50 \mathrm{~g} / \mathrm{ml}$ and $0.25 \mathrm{~g} / \mathrm{ml})$ were prepared by dissolving in $4 \%$ Dimethyl sulphoxide (DMSO). These concentrations were put in sterile bijou bottles and refrigerated at $4^{0} \mathrm{C}$ during use.

\section{Bacterial Strains}

Four strains with American Type Culture Collection numbers (ATCC) were used; two Gram negative bacteria (E. coli ATCC 29211 and P. aeruginosa ATCC 27853) and two Gram positive bacteria (S. aureus ATCC 25923 and MRSA ATCC 43300). These were obtained from archived cultures at the Pharmacy, Complementary and Alternative Medicine departmental laboratories, Kenyatta University. Clinical isolates of E. coli, S. aureus and P. aeruginosa were gifted to me by Mr. Jonathan Mateba of the department of Biochemistry, Microbiology and Biotechnology, Kenyatta University.

\section{Determination of Zones of Inhibition, Minimum Inhibitory Concentration and Minimum Bactericidal Concentration}

Antibacterial assays were done according to the Clinical and Laboratory Standards Institute guidelines [18]. Assays were done in the Medical Laboratory Sciences departmental laboratories at Kenyatta 
University. The test bacteria were sub-cultured on nutrient agar medium, and incubated at $37^{\circ} \mathrm{C}$ for 18 hours. Using a sterile wire loop, fresh growing colonies were transferred into capped sterile glass tubes containing nutrient broth and incubated at $37^{\circ} \mathrm{C}$ for 18 hours to form the stock suspensions.

Microbial cultures from the stock suspensions were inoculated onto the surface of Mueller Hinton Agar and incubated for $18 \mathrm{hrs}$ to obtain fresh growing colonies. A fresh growing bacterial colony was picked from a petri dish using a sterilized wire loop, inoculated into $4 \mathrm{ml}$ of sterile peptone water and incubated at $37^{\circ} \mathrm{C}$ for $4 \mathrm{hrs}$. The bacterial suspensions were then adjusted in order to obtain turbidity comparable to 0.5 McFarland's standard, which corresponds to about $1-2 \times 10^{8}$ colony forming units $/ \mathrm{ml}$. These suspensions were used within fifteen minutes of preparation.

Determination of antibacterial susceptibility patterns was done by the agar disc diffusion method as per Njeru et al. with slight modifications [19]. Gentamycin $(40 \mathrm{mcg} / \mathrm{ml})$ and Neomycin $(200 \mathrm{mcg} / \mathrm{ml})$ were used as positive controls. Paper discs were prepared from a Whatman filter paper, placed in bijou bottles and sterilized. Aliquots of $0.1 \mathrm{~mL}$ of the bacterial suspensions were aseptically pipetted and inoculated by spread plate method on the Mueller hinton agar. The paper discs were impregnated with $20 \mu \mathrm{l}$ of each concentration of the plant extract and control antibiotics and placed on the surface of the inoculated petri dishes using sterile forceps. Dimethyl sulphoxide at $4 \%$ was used as the negative control. Each disk was pressed against the agar medium to ensure level and complete contact. The agar plates were inverted and incubated at $37^{\circ} \mathrm{C}$ for $24 \mathrm{hrs}$. After the specified time, diameters of zones of inhibition formed around discs were measured in millimeters $(\mathrm{mm})$ to determine the activity of test samples against different strains. The scale used for determining the strength of antibacterial activities was 8-13 mm: low inhibition; 14-19 mm: moderate inhibition; $\geq 20 \mathrm{~mm}$ : high inhibition [20].

\section{Determination of Minimum Inhibitory Concentrations and Minimum Bactericidal Concentration}

The broth microdilution method [21] was used to determine the minimum inhibitory concentrations (MIC) and the minimum bactericidal concentrations (MBC). A bacterial colony of each test bacterium was picked using a sterilized wire loop and placed into $4 \mathrm{ml}$ of Mueller Hinton broth medium in test tubes and incubated at $37^{\circ} \mathrm{C}$ for 4 hours. The bacterial suspensions were then adjusted to $0.5 \mathrm{McF}$ arland's standard. One hundred $\mu$ aliquots of the inoculated broth at McFarland's standard were placed into each well of the 96 well microtiter plates. One hundred microliters of the $1 \mathrm{~g} / \mathrm{ml}$ concentration of the extract was pipetted and added into each of the first well of the inoculated microtiter plate and serial diluted eight times. The extract dilutions obtained ranged from $1 \mathrm{~g} / \mathrm{ml}$, to $3.90624 \mathrm{mg} / \mathrm{ml}$. One hundred microliters of gentamycin were pipetted into the control wells and serial diluted to concentrations that ranged from $20 \mathrm{mcg} / \mathrm{ml}$ to $0.15625 \mathrm{mcg} / \mathrm{ml}$. Serial dilutions of neomycin were also made. Dimethyl sulphoxide was used as the negative control. The microtiter plates were incubated at $37^{\circ} \mathrm{C}$ for 24 hours. The concentrations that inhibited growth were taken as the MIC.

For MBC determination, fifty $\mu \mathrm{l}$ aliquots from each inhibited well were pipetted and sero-diluted to the ninth dilution. Fifty $\mu$ l aliquots of the ninth dilution were pipetted and inoculated onto the surface of the 
agar plates. Fifty $\mu \mathrm{l}$ aliquots of the bacterial suspensions in the wells immediately above and below the MIC well were also serial-diluted and inoculated onto the surface of the agar plates. The inoculated plates were incubated at $37^{\circ} \mathrm{C}$ for $24 \mathrm{hrs}$. The lowest concentrations that showed complete inhibition of bacterial growth were taken as the minimum bactericidal concentrations.

\section{Data Analysis}

The antibacterial experiments were done in triplicates. Statistical analyses were done by One way analysis of variance (ANOVA) and students T-test. Data is presented as Means \pm Standard deviation from the mean. Significance was set at $p<0.05$. Statistical analyses were carried out using Minitab version 17 .

\section{Results}

\section{Percentage Yield}

The percentage yield of $O$. basilicum ethyl acetate leaf extract used in the study was $3.2 \%$

Percentage Yield $=\frac{16.02}{500} \times 100=3.2 \%$

Phytochemical Concentration in the Ethyl Acetate Leaf Extract of O. basilicum

The GC-MS analysis of the ethyl acetate leaf extract of 0 . basilicum revealed the presence of 30 compounds. Based on the obtained results, Nootkatone, a sesquiterpenoid had the highest concentration at $20.86 \mathrm{mg} / \mathrm{g}$. The compound 1,3-Dimethyl-5-isobutylcyclohexane, a fatty acid had the lowest concentration at $0.10 \mathrm{mg} / \mathrm{g}$. The retention time, molecular formula, the chemical class and the concentrations $(\mathrm{mg} / \mathrm{g})$ of the identified compounds in Ocimum basilicum leaf extract are presented in Table 1 and Fig. 1 below. 
Table 1

Phytochemical Concentration in the Ethyl Acetate Leaf Extract of 0 . basilicum

\begin{tabular}{|c|c|c|c|c|}
\hline $\begin{array}{l}\text { RT } \\
(\min )\end{array}$ & Phytochemical compound & $\begin{array}{l}\text { Molecular } \\
\text { Formula }\end{array}$ & $\begin{array}{l}\text { Chemical } \\
\text { Class }\end{array}$ & $\begin{array}{l}\text { Concentration } \\
(\mathrm{mg} / \mathrm{g})\end{array}$ \\
\hline 4.9188 & Oxirane, 2-methyl-2-(1-methylethyl)- & $\mathrm{C}_{6} \mathrm{H}_{12} \mathrm{O}$ & Fatty acid & 0.12 \\
\hline 6.6882 & $\begin{array}{l}\text { 9-Thiabicyclo[3.3.1]non-6-en-2- } \\
\text { amine, N-methyl-, endo- }\end{array}$ & $\mathrm{C}_{9} \mathrm{H}_{15} \mathrm{NS}$ & Amine & 0.26 \\
\hline 8.6367 & $\begin{array}{l}\text { 1H-Imidazole, 2-ethyl-4,5-dihydro-4- } \\
\text { methyl- }\end{array}$ & $\mathrm{C}_{6} \mathrm{H}_{12} \mathrm{~N}_{2}$ & Phenol & 0.47 \\
\hline 8.9055 & 1,3-Dimethyl-5-isobutylcyclohexane & $\mathrm{C}_{12} \mathrm{H}_{24}$ & Fatty acid & 0.10 \\
\hline 9.4654 & 3,6,6-Trimethyl-cyclohex-2-enol & $\mathrm{C}_{9} \mathrm{H}_{16} \mathrm{O}$ & Phenols & 1.96 \\
\hline 9.6894 & $\begin{array}{l}\text { 1-Butanol, 3-methyl-, carbonate (2:1) } \\
\text { (Diisopentyl carbonate) }\end{array}$ & $\mathrm{C}_{11} \mathrm{H}_{22} \mathrm{O}_{3}$ & Ester & 0.27 \\
\hline 11.2572 & $\begin{array}{l}\text { 9,10-Dehydro-6-desoxy- } \\
\text { indolinocodeine }\end{array}$ & $\mathrm{C}_{18} \mathrm{H}_{19} \mathrm{NO}_{2}$ & Alkaloids & 0.14 \\
\hline 20.1489 & Tetradecanal & $\mathrm{C}_{14} \mathrm{H}_{28} \mathrm{O}$ & Aldehydes & 3.10 \\
\hline 22.3886 & Tridecenol < 2E-> & $\mathrm{C}_{13} \mathrm{H}_{26} \mathrm{O}$ & Aldehydes & 2.15 \\
\hline 22.7021 & $\begin{array}{l}\text { 5-Aminomethyl-5-oxo-1,3,5- } \\
\text { diazaphosphorinane }\end{array}$ & $\mathrm{C}_{14} \mathrm{H}_{17} \mathrm{~N}_{3} \mathrm{O}_{4}$ & Amine & 0.65 \\
\hline 24.4939 & Manool oxide & $\mathrm{C}_{20} \mathrm{H}_{34} \mathrm{O}$ & Diterpene & 0.57 \\
\hline 25.5018 & 2-Methoxyamphetamine & $\mathrm{C}_{10} \mathrm{H}_{15} \mathrm{NO}$ & Amphetamine & 0.15 \\
\hline 26.2185 & $\begin{array}{l}\text { E-11(13-Methyl)tetradecen-1-ol } \\
\text { acetate }\end{array}$ & $\mathrm{C}_{17} \mathrm{H}_{32} \mathrm{O}_{2}$ & Aldehyde & 0.34 \\
\hline 26.9352 & $\begin{array}{l}\text { 2,4,6-Cycloheptatrien-1-one, 3,5-bis- } \\
\text { trimethylsilyl- }\end{array}$ & $\mathrm{C}_{13} \mathrm{H}_{22} \mathrm{OSi}_{2}$ & & 0.18 \\
\hline 27.4503 & 11-Eicosenoic acid, methyl ester & $\mathrm{C}_{21} \mathrm{H}_{40} \mathrm{O}_{2}$ & Fatty acid & 1.73 \\
\hline 29.1749 & 6,3'-Dimethoxyflavone & $\mathrm{C}_{17} \mathrm{H}_{14} \mathrm{O}_{4}$ & Flavanoids & 1.82 \\
\hline 29.9364 & $\begin{array}{l}\text { 1,2,4-Triazol-3-amine, } 5-(1,3,5- \\
\text { trimethyl-4-pyrazolyl)amino- }\end{array}$ & $\mathrm{C}_{8} \mathrm{H}_{13} \mathrm{~N}_{7}$ & & 1.40 \\
\hline 30.6531 & Benzo[h]quinoline, 2,4-dimethyl- & $\mathrm{C}_{15} \mathrm{H}_{13} \mathrm{~N}$ & Alkaloid & 5.77 \\
\hline 31.1235 & $\begin{array}{l}\text { 2,6,10,14,18-Pentamethyl- } \\
2,6,10,14,18 \text {-eicosapentaene }\end{array}$ & $\mathrm{C}_{25} \mathrm{H}_{42}$ & Triterpene & 2.69 \\
\hline
\end{tabular}




\begin{tabular}{|c|c|c|c|c|}
\hline $\begin{array}{l}\text { RT } \\
(\min )\end{array}$ & Phytochemical compound & $\begin{array}{l}\text { Molecular } \\
\text { Formula }\end{array}$ & $\begin{array}{l}\text { Chemical } \\
\text { Class }\end{array}$ & $\begin{array}{l}\text { Concentration } \\
(\mathrm{mg} / \mathrm{g})\end{array}$ \\
\hline 32.0418 & $\begin{array}{l}\text { Benzothiophene-3-carboxylic acid, } \\
\text { 4,5,6,7-tetrahydro-2-amino-6-ethyl-, } \\
\text { ethyl ester }\end{array}$ & $\mathrm{C}_{18} \mathrm{H}_{18} \mathrm{ClNO}_{3} \mathrm{~S}$ & Ester & 1.44 \\
\hline 34.3487 & Eicosane, 10-heptyl-10-octyl- & $\mathrm{C}_{35} \mathrm{H}_{72}$ & Essential oils & 3.19 \\
\hline 35.8269 & $\begin{array}{l}\text { 1H-Pyrazole, 1-(3-methylbutyl)-4- } \\
\text { (4,4,5,5-tetramethyl-1,3,2- } \\
\text { dioxaborolan-2-yl)- }\end{array}$ & $\mathrm{C}_{14} \mathrm{H}_{25} \mathrm{BN}_{2} \mathrm{O}_{2}$ & Esters & 2.92 \\
\hline 39.724 & .beta.-Amyrin & $\mathrm{C}_{30} \mathrm{H}_{50} \mathrm{O}$ & Triterpene & 7.75 \\
\hline 40.2391 & $\begin{array}{l}\text { 1,2,4,8- } \\
\text { Tetramethylbicyclo[6.3.0]undeca-2,4- } \\
\text { diene (Isocaryophyllene) }\end{array}$ & $\mathrm{C}_{15} \mathrm{H}_{24}$ & Sesquiterpene & 6.72 \\
\hline 40.7543 & $\begin{array}{l}\text { 2(1H)Naphthalenone, } 3,5,6,7,8,8 \mathrm{a}- \\
\text { hexahydro-4,8a-dimethyl-6-(1- } \\
\text { methylethenyl)-(Nootkatone) }\end{array}$ & $\mathrm{C}_{15} \mathrm{H}_{22} \mathrm{O}$ & Sesquiterpene & 20.86 \\
\hline 42.1429 & Zierone & $\mathrm{C}_{15} \mathrm{H}_{22} \mathrm{O}$ & Sesquiterpene & 11.45 \\
\hline 42.882 & $\begin{array}{l}\text { 3-Quinolinecarboxylic acid, 6,8- } \\
\text { difluoro-4-hydroxy-, ethyl ester }\end{array}$ & $\mathrm{C}_{12} \mathrm{H}_{9} \mathrm{~F}_{2} \mathrm{NO}_{3}$ & Alkaloid & 0.93 \\
\hline 43.0612 & $\begin{array}{l}\text { 9,10-Methanoanthracen-11-ol, 9,10- } \\
\text { dihydro-9,10,11-trimethyl- }\end{array}$ & $\mathrm{C}_{18} \mathrm{H}_{18} \mathrm{O}$ & Phenol & 0.60 \\
\hline 43.3075 & $\begin{array}{l}\text { A'-Neogammacer-22(29)-en-3-ol, } \\
\text { acetate, (3.beta.,21.beta.)- (Lupeol } \\
\text { acetate) }\end{array}$ & $\mathrm{C}_{32} \mathrm{H}_{52} \mathrm{O}_{2}$ & Triterpene & 16.76 \\
\hline 46.7119 & $\begin{array}{l}\text { I-Alanine, N-(2-thienylacetyl)-, butyl } \\
\text { ester }\end{array}$ & $\mathrm{C}_{9} \mathrm{H}_{14} \mathrm{~F}_{3} \mathrm{NO}_{3}$ & Phenol & 0.25 \\
\hline
\end{tabular}

\section{Antibacterial Activities of the Ethyl Acetate Leaf Extract of O. basilicum}

The antibacterial activities of the extract are presented in Tables 2-6. A broad spectrum antibacterial activity was exhibited by the extract of $O$. basilicum. The zones of inhibition ranged from $17.33 \pm$ $0.58 \mathrm{~mm}$ to $27.00 \pm 2.00 \mathrm{~mm}$ in diameter. Gram negative bacteria showed more susceptibility to the organic extract compared to the gram positive bacteria. Among the gram negatives, $P$. aeruginosa had the highest zone of inhibition $(27.00 \pm 2.00 \mathrm{~mm})$, while among the gram positives, MRSA had the highest zone of inhibition $(25.00 \pm 1.73 \mathrm{~mm})$ at $1 \mathrm{~g} / \mathrm{ml}$ concentration of the organic extracts. There was a gradual decrease in the antibacterial activity of the extract that coincided with a decrease in the concentration of the extract. 
Table 2

An Antibiogram of Bacterial Strains

\begin{tabular}{|c|c|c|c|c|c|c|c|}
\hline \multicolumn{8}{|c|}{ Zones of Inhibition (mm) } \\
\hline Test Agents & $\begin{array}{l}\text { MRSA } \\
\text { (s) }\end{array}$ & $\begin{array}{l}S . \\
\text { aureus } \\
(s)\end{array}$ & $\begin{array}{l}S . \\
\text { aureus } \\
\text { (i) }\end{array}$ & $\begin{array}{l}\text { E. coli } \\
(\mathrm{s})\end{array}$ & E. coli $(\mathrm{i})$ & $\begin{array}{l}P . \\
\text { aeruginosa } \\
\text { (s) }\end{array}$ & $\begin{array}{l}\text { P. } \\
\text { aeruginosa } \\
\text { (i) }\end{array}$ \\
\hline Gentamycin & $\begin{array}{l}32.33 \pm \\
2.08^{\mathrm{a}}\end{array}$ & $\begin{array}{l}33.00 \pm \\
1.00^{\mathrm{a}}\end{array}$ & $\begin{array}{l}29.00 \pm \\
1.00^{\mathrm{a}}\end{array}$ & $\begin{array}{l}32.00 \pm \\
1.00^{\mathrm{a}}\end{array}$ & $\begin{array}{l}27.00 \pm \\
1.00^{\mathrm{a}}\end{array}$ & $\begin{array}{l}31.33 \pm \\
1.53^{\mathrm{a}}\end{array}$ & $\begin{array}{l}28.67 \pm \\
1.53^{\mathrm{a}}\end{array}$ \\
\hline Neomycin & $\begin{array}{l}6.00 \pm \\
00^{d}\end{array}$ & $\begin{array}{l}27.67 \pm \\
0.58^{b}\end{array}$ & $\begin{array}{l}13.33 \pm \\
0.58^{\mathrm{e}}\end{array}$ & $\begin{array}{l}31.33 \pm \\
0.58^{\mathrm{a}}\end{array}$ & $\begin{array}{l}15.33 \pm \\
0.58^{\mathrm{e}}\end{array}$ & $\begin{array}{l}27.00 \pm \\
1.00^{\mathrm{b}}\end{array}$ & $\begin{array}{l}14.33 \pm \\
2.08^{\mathrm{c}}\end{array}$ \\
\hline DMSO & $\begin{array}{l}6.00 \pm \\
0.00^{d}\end{array}$ & $\begin{array}{l}6.00 \pm \\
0.00^{f}\end{array}$ & $\begin{array}{l}6.00 \pm \\
0.00^{f}\end{array}$ & $\begin{array}{l}6.00 \pm \\
0.00^{d}\end{array}$ & $\begin{array}{l}6.00 \pm \\
0.00^{f}\end{array}$ & $\begin{array}{l}6.00 \pm \\
0.00^{d}\end{array}$ & $\begin{array}{l}6.00 \pm \\
0.00^{d}\end{array}$ \\
\hline $\begin{array}{l}1 \mathrm{~g} / \mathrm{ml} \\
\text { Extract }\end{array}$ & $\begin{array}{l}25.00 \pm \\
1.73^{\mathrm{b}}\end{array}$ & $\begin{array}{l}24.68 \pm \\
0.58^{\mathrm{c}}\end{array}$ & $\begin{array}{l}24.00 \pm \\
1.00^{\mathrm{b}}\end{array}$ & $\begin{array}{l}26.00 \pm \\
1.00^{\mathrm{b}}\end{array}$ & $\begin{array}{l}24.68 \pm \\
0.58^{\mathrm{ab}}\end{array}$ & $\begin{array}{l}27.00 \pm \\
2.00^{\mathrm{b}}\end{array}$ & $\begin{array}{l}26.67 \pm \\
1.53^{\mathrm{a}}\end{array}$ \\
\hline $\begin{array}{l}0.75 \mathrm{~g} / \mathrm{ml} \\
\text { Extract }\end{array}$ & $\begin{array}{l}23.33 \pm \\
1.53^{b}\end{array}$ & $\begin{array}{l}23.00 \pm \\
1.00^{c}\end{array}$ & $\begin{array}{l}21.68 \pm \\
1.53^{b c}\end{array}$ & $\begin{array}{l}24.68 \pm \\
1.53^{b}\end{array}$ & $\begin{array}{l}22.68 \pm \\
1.53^{b c}\end{array}$ & $\begin{array}{l}23.33 \pm \\
0.58 \mathrm{bc}\end{array}$ & $\begin{array}{l}21.67 \pm \\
1.56^{\mathrm{b}}\end{array}$ \\
\hline $\begin{array}{l}0.50 \mathrm{~g} / \mathrm{ml} \\
\text { Extract }\end{array}$ & $\begin{array}{l}22.00 \pm \\
1.00^{b}\end{array}$ & $\begin{array}{l}21.68 \pm \\
0.58^{d}\end{array}$ & $\begin{array}{l}20.33 \pm \\
1.53^{c}\end{array}$ & $\begin{array}{l}22.67 \pm \\
2.52^{b c}\end{array}$ & $\begin{array}{l}21.33 \pm \\
1.53^{c d}\end{array}$ & $\begin{array}{l}22.33 \pm \\
2.52^{c}\end{array}$ & $\begin{array}{l}21.33 \pm \\
1.53^{\mathrm{b}}\end{array}$ \\
\hline $\begin{array}{l}0.25 \mathrm{~g} / \mathrm{ml} \\
\text { Extract }\end{array}$ & $\begin{array}{l}18.00 \pm \\
0.00^{c}\end{array}$ & $\begin{array}{l}18.00 \pm \\
1.00^{\mathrm{e}}\end{array}$ & $\begin{array}{l}17.33 \pm \\
0.58^{d}\end{array}$ & $\begin{array}{l}20.00 \pm \\
1.00^{c}\end{array}$ & $\begin{array}{l}18.67 \pm \\
1.53^{d}\end{array}$ & $\begin{array}{l}19.67 \pm \\
0.58^{\mathrm{c}}\end{array}$ & $\begin{array}{l}18.67 \pm \\
1.53^{\mathrm{b}}\end{array}$ \\
\hline
\end{tabular}

\section{Two Sample T tests for the determination of Significance differences between strains}

The clinical isolates exhibited a reduced susceptibility to the organic extract compared to the quality control strains. However, this difference was not significant between the ATCC strains and the clinical isolates. A notable reduction in the zones of inhibition was observed in tandem with decreasing concentrations of the extract. These results are presented in Tables 3, 4 and 5. 
Table 3

An Antibiogram of $S$. aureus

\section{Zones of inhibition (mm)}

\begin{tabular}{|llll|}
\hline Test Agents & S. aureus $(\mathrm{s})$ & S. aureus $(\mathrm{i})$ & p. Values \\
\hline Gentamycin & $33.00 \pm 1.00$ & $29.00 \pm 1.00$ & 0.008 \\
\hline Neomycin & $27.67 \pm 0.58$ & $13.33 \pm 0.58$ & 0.000 \\
\hline $1 \mathrm{~g} / \mathrm{ml}$ Extract & $24.68 \pm 0.58$ & $24.00 \pm 1.00$ & 0.391 \\
\hline $0.75 \mathrm{~g} / \mathrm{ml}$ Extract & $23.00 \pm 1.00$ & $21.68 \pm 1.53$ & 0.295 \\
\hline $0.50 \mathrm{~g} / \mathrm{ml}$ Extract & $21.68 \pm 0.58$ & $20.33 \pm 1.53$ & 0.293 \\
\hline $0.25 \mathrm{~g} / \mathrm{ml}$ Extract & $18.00 \pm 1.00$ & $17.33 \pm 0.58$ & 0.391 \\
\hline Values are expressed as Mean \pm S.D for $\mathrm{n}=3$. Key: $\mathrm{s}-$ ATCC strains, $\mathrm{i}-$ clinical isolate strains.
\end{tabular}

Table 4

An Antibiogram of E. coli

\section{Zones of Inhibition (mm)}

\begin{tabular}{|llll|}
\hline Test Agents & E. coli $(\mathrm{s})$ & E. coli $(\mathrm{i})$ & p. values \\
\hline Gentamycin & $32.00 \pm 1.00$ & $27.00 \pm 1.00$ & 0.004 \\
\hline Neomycin & $31.33 \pm 0.58$ & $15.33 \pm 0.58$ & 0.000 \\
\hline $1 \mathrm{~g} / \mathrm{ml}$ Extract & $26.00 \pm 1.00$ & $24.68 \pm 0.58$ & 0.139 \\
\hline $0.75 \mathrm{~g} / \mathrm{ml}$ Extract & $24.68 \pm 1.53$ & $22.68 \pm 1.53$ & 0.184 \\
\hline $0.50 \mathrm{~g} / \mathrm{ml}$ Extract & $22.67 \pm 2.52$ & $21.33 \pm 1.53$ & 0.490 \\
\hline $0.25 \mathrm{~g} / \mathrm{ml}$ Extract & $20.00 \pm 1.00$ & $18.67 \pm 1.53$ & 0.295 \\
\hline Values are expressed as Mean \pm S.D for $\mathrm{n}=3$. Key: $\mathrm{s}-$ ATCC strains, $\mathrm{i}-$ clinical isolate strains. \\
\hline
\end{tabular}


Table 5

An Antibiogram of $P$. aeruginosa

\begin{tabular}{|llll|}
\hline Zones of Inhibition $(\mathrm{mm})$ & & \\
\hline Test Agent & $P$. aeruginosa $(\mathrm{s})$ & $P$. aeruginosa $(\mathrm{i})$ & p. values \\
\hline Gentamycin & $31.33 \pm 1.53$ & $28.67 \pm 1.53$ & 0.099 \\
\hline Neomycin & $27.00 \pm 1.00$ & $14.33 \pm 2.08$ & 0.011 \\
\hline $1 \mathrm{~g} / \mathrm{ml}$ Extract & $27.00 \pm 2.00$ & $26.67 \pm 1.53$ & 0.833 \\
\hline $0.75 \mathrm{~g} / \mathrm{ml}$ Extract & $23.33 \pm 0.58$ & $21.67 \pm 1.56$ & 0.155 \\
\hline $0.50 \mathrm{~g} / \mathrm{ml}$ Extract & $22.33 \pm 2.52$ & $21.33 \pm 1.53$ & 0.598 \\
\hline $0.25 \mathrm{~g} / \mathrm{ml}$ Extract & $19.67 \pm 0.58$ & $18.67 \pm 1.53$ & 0.400 \\
\hline Values are expressed as Mean \pm S.D for $\mathrm{n}=3$. Key: $\mathrm{s}-$ ATCC strains, $\mathrm{i}-$ clinical isolate strains.
\end{tabular}

\section{Minimum Inhibitory Concentration and Minimum Bactericidal Concentration}

At a concentration of $62.5 \mathrm{mg} / \mathrm{ml}$, the extract exhibited its MIC on E. coli, S. aureus and MRSA while on $P$. aeruginosa, MIC was manifested at a concentration of $125 \mathrm{mg} / \mathrm{ml}$. The leaf extract exhibited an MBC of $62.5 \mathrm{mg} / \mathrm{ml}$ on the $S$. aureus strain and an MBC of $125 \mathrm{mg} / \mathrm{ml}$ on MRSA, E. coli and P. aeruginosa. These results are presented in Table 6 below.

Table 6

MIC and MBC Values of Different Antimicrobial Agents on Selected Bacterial Strains

\begin{tabular}{|c|c|c|c|c|c|c|}
\hline & MIC (g/ml) & & & $\mathrm{MBC}(\mathrm{g} / \mathrm{ml})$ & & \\
\hline Pathogen & Gentamycin & Extract & Neomycin & Gentamycin & Extract & Neomycin \\
\hline MRSA & 0.156 & 62.5 & - & 0.156 & 125.0 & - \\
\hline S. aureus (s) & 0.156 & 62.5 & 12.5 & 0.156 & 62.5 & 25.0 \\
\hline S. aureus (i) & 0.156 & 62.5 & 50.0 & 0.312 & 62.5 & 100.0 \\
\hline E. coli (s) & 0.312 & 62.5 & 12.5 & 0.312 & 125.0 & 25.0 \\
\hline E. coli (i) & 0.312 & 62.5 & 50.0 & 0.625 & 125.0 & 100.0 \\
\hline P. aeruginosa (s) & 0.312 & 125.0 & 12.5 & 0.312 & 125.0 & 25.0 \\
\hline P. aeruginosa (i) & 0.312 & 125.0 & 50.0 & 1.250 & 125.0 & 100.0 \\
\hline
\end{tabular}




\section{Discussion}

Medicinal plants contribute to the development of new chemo-preventive agents. It is therefore important to determine their bioactive compounds as well as antibacterial activities [22]. Plants produce a wide collection of phytochemicals that are related to stress, defence mechanisms and antimicrobial activities [23]. The ethyl acetate leaf extract of $O$. basilicum had a variety of phytochemical classes and exhibited antibacterial activities.

Flavonoids are free radical scavengers and water soluble antioxidants with the ability to prevent oxidative cell damage. These metabolites protect the body against cancer, inflammation, allergens, microbes, platelet aggregation, tumors and hepatotoxins [24]. The methylated flavonoid, 6,3'-Dimethoxyflavone exhibits antimicrobial activities. Its derivative has broad spectrum antibacterial activities on $E$. coli, $P$. aeruginosa and $S$. aureus $[25,26]$.

The two alkaloids 3-Quinolinecarboxylic acid, 6,8-difluoro-4-hydroxy-, ethyl ester and Benzo[h]quinoline, 2,4-dimethyl-. Benzo[h]quinolones have been used to obtain novel compounds that possess antibacterial activities on S. aureus, Bacillus subtilis, and Streptococcus pyogenes. These compounds are also strong antioxidants, show significant wound healing activities and protect oxidative DNA damage from harmful free radical reactions [27]. Synthetic derivatives of alkaloids are medicinally important for their antispasmodic, analgesic and bactericidal effects. Its physiological activities are apparent when this metabolite is administered in animal models [28].

Terpenoids represent the most diverse and largest class of chemicals among the many metabolites produced by plants. They are a group of compounds possessing an isoprene unit as their basic structure. They are classified based on the number of carbon atoms. Terpenoids provide protection against pathogenic microorganisms [29]. Nootkatone is a sesquiterpene that is synthesized by the oxidation of valencene [30]. Nootkatone has been shown to exhibit antibacterial activities on Gram-positive bacteria including S. aureus, Enterococcus faecalis, Corynebacterium diphtheriae, Listeria monocytogenes and Bacillus cereus [30]. This compound exhibits antibacterial effects by targeting metabolites or structures that are specific to Gram-positive bacteria such as the peptidoglycan component of cell walls. Synthetic retinoids contain an isoprene unit that is capable of killing MRSA by penetrating and disrupting the lipid bilayers [31]. There is a possibility that nootkatone inhibits bacterial proliferation by acting on the synthetic pathway of peptidoglycan.

$\beta$-caryophyllene is a sesquiterpene, component of dietary regimes that is consumed as a food preservative, flavour or additive and is often referred to as a 'dietary cannabinoid' [32]. $\beta$-caryophyllene has exhibited therapeutic potential due to multiple pharmacological properties such as antioxidant, antiinflammatory [32] and antimicrobial [33]. Triterpenoids such as $\beta$-amyrin, a pentacyclic triterpene, are plant constituents with great pharmacological potentials [34]. Lupeol acetate, a pentacyclic triterpene, exhibits a wide spectrum of pharmacological effects including antimicrobial effects [35]. The triterpene 2,6,10,14,18-Pentamethyl-2,6,10,14,18-eicosapentaene was identified in the ethyl acetate leaf extract of 
Ocimum basilicum. Manool oxide, a diterpene, has been found to be in abundance in several essential oils, from several plants, which have been shown to have antibacterial activities [36, 37].

Fatty acids identified include Oxirane, 2-methyl-2-(1-methylethyl)-; 11-Eicosenoic acid, methyl ester and 1,3-Dimethyl-5-isobutylcyclohexane. 11-Eicosenoic acid, methyl ester has been associated with antibacterial activity and anti-inflammatory effects [38]. The exact mechanisms by which fatty acids impose their antibacterial effects remain unknown. It has, however, been hypothesized that these molecules induce peroxidative processes that inhibit bacterial fatty acid synthesis. Fatty acids may also interact with cellular membranes thereby causing leakage of molecules from the cells, reduction of nutrient uptake or inhibiting cellular respiration [42]. Amines eluted from the extract include 9-Thiabicyclo [3.3.1] non-6-en-2-amine, N-methyl-, endo-; 1,2,4-Triazol-3-amine, 5-(1,3,5-trimethyl-4-pyrazolyl) amino- and 2-Methoxyamphetamine. Bicyclic amines have been documented to have antimicrobial and antiparasitic effects $[39,40]$. Aldehydes with long chain fatty alcohols such as E-11(13-Methyl) tetradecen-1-ol acetate, Tridecenol <2E-> and tetradecanal have been shown to have antibacterial activities on $S$. aureus [41]. Tetradecanaal and tridecanol are long chain alcohols that exhibit their antibacterial activity by damaging cell membranes thereby leading to the leakage of $\mathrm{K}+$ ions together with subsequent reactions that lead to further leakage [41]. Compounds that possess an alkyl chain promote antibacterial activity and resensitize methicillin susceptible and resistant $S$. aureus to antibiotics [41].

Phenolic compounds with imidazole moieties such as 1H-Imidazole, 2-ethyl-4,5-dihydro-4-methyl-; 3,6,6Trimethyl-cyclohex-2-enol and I-Alanine, N-(2-thienylacetyl)-, butyl ester possess antibacterial properties. The presence of the imidazole ring to the quinolone moiety increases its antibacterial activity [43]. The antibacterial effects exhibited by the extract were broad spectrum and could be attributed to the effects of these phytochemicals. The inhibitory effects were exhibited in a dose dependent manner with a better efficacy being exhibited on the gram positive bacteria compared to the gram negative. The difference in susceptibility indices could be attributed to the fact that gram negative bacteria have a stable peptidoglycan layer that allows bioactive compounds into the cytoplasm at a lower rate compared to the gram positive bacteria. The dose dependent active nature of the extract on the bacterial strains was due to the decreasing concentrations of active compounds. The ethyl acetate leaf extract of 0 . basilicum was found to have strong antibacterial properties [20].

\section{Conclusions}

The results of this study provide an important basis for the use $O$. basilicum in the treatment of bacterial diseases. The extract also contained various pharmacologically active compounds that could be used as lead molecules in the development of new antibacterials. The presence of active chemical groups such as flavonoids, triterpenes, phenols and others in the leaves of $O$. basilicum justify the results obtained. This study also provides an important basis for the use of ethyl acetate extract of $O$. basilicum to control infectious diseases

\section{List Of Abbreviations}


O. basilicum Ocimum basilicum

S.aureus Staphylococcus aureus

$P$. aeruginosa Pseudomonas aeruginosa

E. coli Escherichia coli

MRSA Methicillin resistant Staphylococcus aureus

DMSO Dimethyl sulphoxide

ATCC American Type Culture Collection

MIC Minimum inhibitory concentration

MBC Minimum bactericidal concentration

ANOVA Analysis of Variance

\section{Declarations}

\section{Ethical approval and consent to participate}

Not applicable.

\section{Consent for publication}

Not applicable

\section{Funding}

Not applicable

\section{Availability of data and materials}

The datasets used and/or analyzed during the current study are available from the corresponding author on reasonable request.

\section{Competing interests}

The authors declare no competing interests

\section{Authors' contributions}

JWR Conceived and designed the study, collected the plant, collected, analysed, interpreted the data and wrote the manuscript. MPN conceived and designed the study, collected the plant, interpreted the data, 
substantively revised the manuscript; MM conceived and designed the study, interpreted the data, substantively revised the manuscript; JOO conceived and designed the study, interpreted the data, substantively revised the manuscript .

\section{Acknowledgements}

The authors acknowledge the assistance of technicians in the departments of Biochemistry, Microbiology and Biotechnology; the department of Medical Laboratory Sciences and the department of Pharmacy. Jonathan Mateba is acknowledged for providing the clinical isolates of the test bacteria.

\section{References}

1. NIE 99-17D NIE. The global infectious disease threat and its implications for the United States. 2000. Retrieved from http://www.heart-intl.net/HEART/072404/ on July 3, 2020.

2. CDC. Antibiotic/Antimicrobial Resistance. Retrieved from https://www.cdc.gov/drugresistance/index.html on 3rd July, 2020

3. World Health Organization (2014). Antimicrobial Resistance: Global Report on Surveillance. Geneva: Retrieved from https://www.who.int/antimicrobial-resistance/publications/surveillancereport/en/ on July 3, 2020.

4. Römling U, Balsalobre C. Biofilm infections, their resilience to therapy and innovative treatment strategies. Journal of internal medicine. 2012 Dec;272(6):541-61.

5. Kim OY, Hong BS, Park KS, Yoon YJ, Choi SJ, Lee WH, Roh TY, Lötvall J, Kim YK, Gho YS. Immunization with Escherichia coli outer membrane vesicles protects bacteria-induced lethality via Th1 and Th17 cell responses. The Journal of Immunology. 2013 Apr 15;190(8):4092-102.

6. Dilnessa T, Bitew A. Prevalence and antimicrobial susceptibility pattern of methicillin resistant Staphylococcus aureus isolated from clinical samples at Yekatit 12 Hospital Medical College, Addis Ababa, Ethiopia. BMC infectious diseases. 2016 Dec 1;16(1):398.

7. Mamuye Y. Antibiotic resistance patterns of common Gram-negative uropathogens in St. Paul's Hospital Millennium Medical College. Ethiopian Journal of Health Sciences. 2016 Mar 30;26(2):93100.

8. Wonnenberg B, Bischoff M, Beisswenger C, Dinh T, Bals R, Singh B, Tschernig T. The role of IL-1 $\beta$ in Pseudomonas aeruginosa in lung infection. Cell and Tissue Research. 2016 May 1;364(2):225-9.

9. Akiyama H, Fujii K, Yamasaki O, Oono T, Iwatsuki K. Antibacterial action of several tannins against Staphylococcus aureus. Journal of antimicrobial chemotherapy. 2001 Oct 1;48(4):487-91.

10. Wintola, O. A., \& Afolayan, A. J. (2015). The antibacterial, phytochemicals and antioxidants evaluation of the root extracts of Hydnora africana Thunb. used as antidysenteric in Eastern Cape Province, South Africa. BMC complementary and alternative medicine, 15(1), 307.

11. Gorlenko CL, Kiselev HY, Budanova EV, Zamyatnin AA, Ikryannikova LN. Plant Secondary Metabolites in the Battle of Drugs and Drug-Resistant Bacteria: New Heroes or Worse Clones of Antibiotics?. 
Antibiotics. 2020 Apr;9(4):170.

12. Malapermal V, Botha I, Krishna SB, Mbatha JN. Enhancing antidiabetic and antimicrobial performance of Ocimum basilicum, and Ocimum sanctum (L.) using silver nanoparticles. Saudi Journal of Biological Sciences. 2017 Sep 1;24(6):1294-305.

13. Antora RA, Salleh RM. Antihyperglycemic effect of Ocimum plants: A short review. Asian Pacific Journal of Tropical Biomedicine. 2017 Aug 1;7(8):755-9.

14. Avetisyan A, Markosian A, Petrosyan M, Sahakyan N, Babayan A, Aloyan S, Trchounian A. Chemical composition and some biological activities of the essential oils from basil Ocimum different cultivars. BMC complementary and alternative medicine. 2017 Dec;17(1):1-8.

15. Kareru PG, Kenji GM, Gachanja AN, Keriko JM, Mungai G. Traditional medicines among the Embu and Mbeere people of Kenya. African Journal of Traditional, Complementary and Alternative Medicines. 2007;4(1):75-86.

16. Sui Y, Li S, Shi P, Wu Y, Li Y, Chen W, Huang L, Yao H, Lin X. Ethyl acetate extract from Selaginella doederleinii Hieron inhibits the growth of human lung cancer cells A549 via caspase-dependent apoptosis pathway. Journal of ethnopharmacology. 2016 Aug 22;190:261-71.

17. Arora S, Saini M. Gas Chromatography Mass Spectrometry profiling in methanolic and ethyl-acetate root and stem extract of Corbichonia decumbens (Forssk.) Exell from Thar Desert of Rajasthan, India. Pharmacognosy Research. 2017 Dec;9(Suppl 1):S48.

18. CLSI. Performance standards for antimicrobial susceptibility testing. Retrieved from https://clsi.org/media/2663/m100ed29_sample.pdf on 6th June, 2018.

19. Njeru SN, Obonyo M, Nyambati S, Ngari S, Mwakubambanya R, Mavura H. Antimicrobial and cytotoxicity properties of the organic solvent fractions of Clerodendrum myricoides (Hochst.) R. Br. ex Vatke: Kenyan traditional medicinal plant. Journal of intercultural ethnopharmacology. 2016 Jun;5(3):226.

20. Ambrosio CM, de Alencar SM, de Sousa RL, Moreno AM, Da Gloria EM. Antimicrobial activity of several essential oils on pathogenic and beneficial bacteria. Ind Crop Prod. 2017;97:128-36.

21. de Oliveira, Y. L. C., Nascimento da Silva, L. C., da Silva, A. G., Macedo, A. J., Araújo, J. M. D., Correia, M. T. D. S., \& Silva, M. V. D. (2012). Antimicrobial activity and phytochemical screening of Buchenavia tetraphylla (Aubl.) RA Howard (Combretaceae: Combretoideae). The Scientific World Journal, 2012.

22. Tona L, Cimanga R, Mesia K, Musuamba C, De Bruyne T, Apers S, Hernans N, Van Miert S, Pieters L, Totté J. In vitro antiplasmodial activity of extracts and fractions from seven medicinal plants used in the Democratic Republic of Congo. J Ethnopharmacol. 2004;93:27-32.

23. Dixon RA. Natural products and plant disease resistance. Nature. 2001;411:843-7.

24. Falcone Ferreyra ML, Rius S, Casati P. Flavonoids: biosynthesis, biological functions, and biotechnological applications. Frontiers in plant science. 2012 Sep 28;3:222.

25. Teffo LS, Aderogba MA, Eloff JN. Antibacterial and antioxidant activities of four kaempferol methyl ethers isolated from Dodonaea viscosa Jacq. var. angustifolia leaf extracts. South African Journal of Botany. 2010 Jan 1;76(1):25-9. 
26. Omosa LK, Amugune B, Ndunda B, Milugo TK, Heydenreich M, Yenesew A, Midiwo JO. Antimicrobial flavonoids and diterpenoids from Dodonaea angustifolia. South African journal of botany. 2014 Mar 1;91:58-62.

27. Naik HR, Naik HS, Naik TR, Naika HR, Gouthamchandra K, Mahmood R, Ahamed BK. Synthesis of novel benzo [h] quinolines: wound healing, antibacterial, DNA binding and in vitro antioxidant activity. European journal of medicinal chemistry. 2009 Mar 1;44(3):981-9.

28. Wirasathien L, Boonarkart C, Pengsuparp T, Suttisri R. Biological Activities of Alkaloids from Pseuduvaria setosa. Pharmaceutical biology. 2006 Jan 1;44(4):274-8.

29. Yadav MK, Chae SW, Im GJ, Chung JW, Song JJ. Eugenol: a phyto-compound effective against methicillin-resistant and methicillin-sensitive Staphylococcus aureus clinical strain biofilms. PLoS One. 2015 Mar 17;10(3):e0119564.

30. Leonhardt RH, Berger RG. Nootkatone. InBiotechnology of Isoprenoids 2014 (pp. 391-404). Springer, Cham.

31. Kim W, Zhu W, Hendricks GL, Van Tyne D, Steele AD, Keohane CE, Fricke N, Conery AL, Shen S, Pan W, Lee K. A new class of synthetic retinoid antibiotics effective against bacterial persisters. Nature. 2018 Apr;556(7699):103-7.

32. Gertsch J, Leonti M, Raduner S, Racz I, Chen JZ, Xie XQ, Altmann KH, Karsak M, Zimmer A. Betacaryophyllene is a dietary cannabinoid. Proceedings of the National Academy of Sciences. 2008 Jul 1;105(26):9099-104.

33. Dahham SS, Tabana YM, Iqbal MA, Ahamed MB, Ezzat MO, Majid AS, Majid AM. The anticancer, antioxidant and antimicrobial properties of the sesquiterpene $\beta$-caryophyllene from the essential oil of Aquilaria crassna. Molecules. 2015 Jul;20(7):11808-29.

34. Nogueira AO, Oliveira YI, Adjafre BL, de Moraes ME, Aragao GF. Pharmacological effects of the isomeric mixture of alpha and beta amyrin from Protium heptaphyllum: a literature review. Fundamental \& clinical pharmacology. 2019 Feb;33(1):4-12.

35. Awolola GV, Koorbanally NA, Chenia H, Shode FO, Baijnath H. Antibacterial and anti-biofilm activity of flavonoids and triterpenes isolated from the extracts of Ficus sansibarica Warb. subsp. sansibarica (Moraceae) extracts. African Journal of Traditional, Complementary and Alternative Medicines. 2014 Jul 9;11(3):124-31.

36. Amri I, Hanana M, Jamoussi B, Hamrouni L. Essential oils of Pinus nigra JF Arnold subsp. laricio Maire: Chemical composition and study of their herbicidal potential. Arabian Journal of Chemistry. 2017 May 1;10:S3877-82.

37. Rosato A, Maggi F, Cianfaglione K, Conti F, Ciaschetti G, Rakotosaona R, Fracchiolla G, Clodoveo ML, Franchini C, Corbo F. Chemical composition and antibacterial activity of seven uncommon essential oils. Journal of EssEntial oil rEsEarch. 2018 Jul 4;30(4):233-43.

38. Pereira DM, Correia-da-Silva G, Valentão P, Teixeira N, Andrade PB. Anti-inflammatory effect of unsaturated fatty acids and ergosta-7, 22-dien-3-ol from Marthasterias glacialis: Prevention of CHOPmediated ER-stress and NF-KB activation. PLoS One. 2014 Feb 13;9(2):e88341. 
39. Weis R, Seebacher W. New bicyclic amines: synthesis and SARs of their action against the causative organisms of malaria and sleeping sickness. Current medicinal chemistry. 2009 Apr 1;16(11):142641.

40. Roig-Molina E, Domenech M, de Gracia Retamosa M, Nácher-Vázquez M, Rivas L, Maestro B, García P, García E, Sanz JM. Widening the antimicrobial spectrum of esters of bicyclic amines: In vitro effect on gram-positive Streptococcus pneumoniae and gram-negative non-typeable Haemophilus influenzae biofilms. Biochimica et Biophysica Acta (BBA)-General Subjects. 2019 Jan 1;1863(1):96104.

41. Togashi N, Shiraishi A, Nishizaka M, Matsuoka K, Endo K, Hamashima H, Inoue Y. Antibacterial activity of long-chain fatty alcohols against Staphylococcus aureus. Molecules. 2007 Feb;12(2):13948.

42. Zheng CJ, Yoo JS, Lee TG, Cho HY, Kim YH, Kim WG. Fatty acid synthesis is a target for antibacterial activity of unsaturated fatty acids. FEBS letters. 2005 Sep 26;579(23):5157-62.

43. Zhang GF, Zhang S, Pan B, Liu X, Feng LS. 4-Quinolone derivatives and their activities against Gram positive pathogens. European Journal of Medicinal Chemistry. 2018 Jan 1;143:710-23.

\section{Figures}



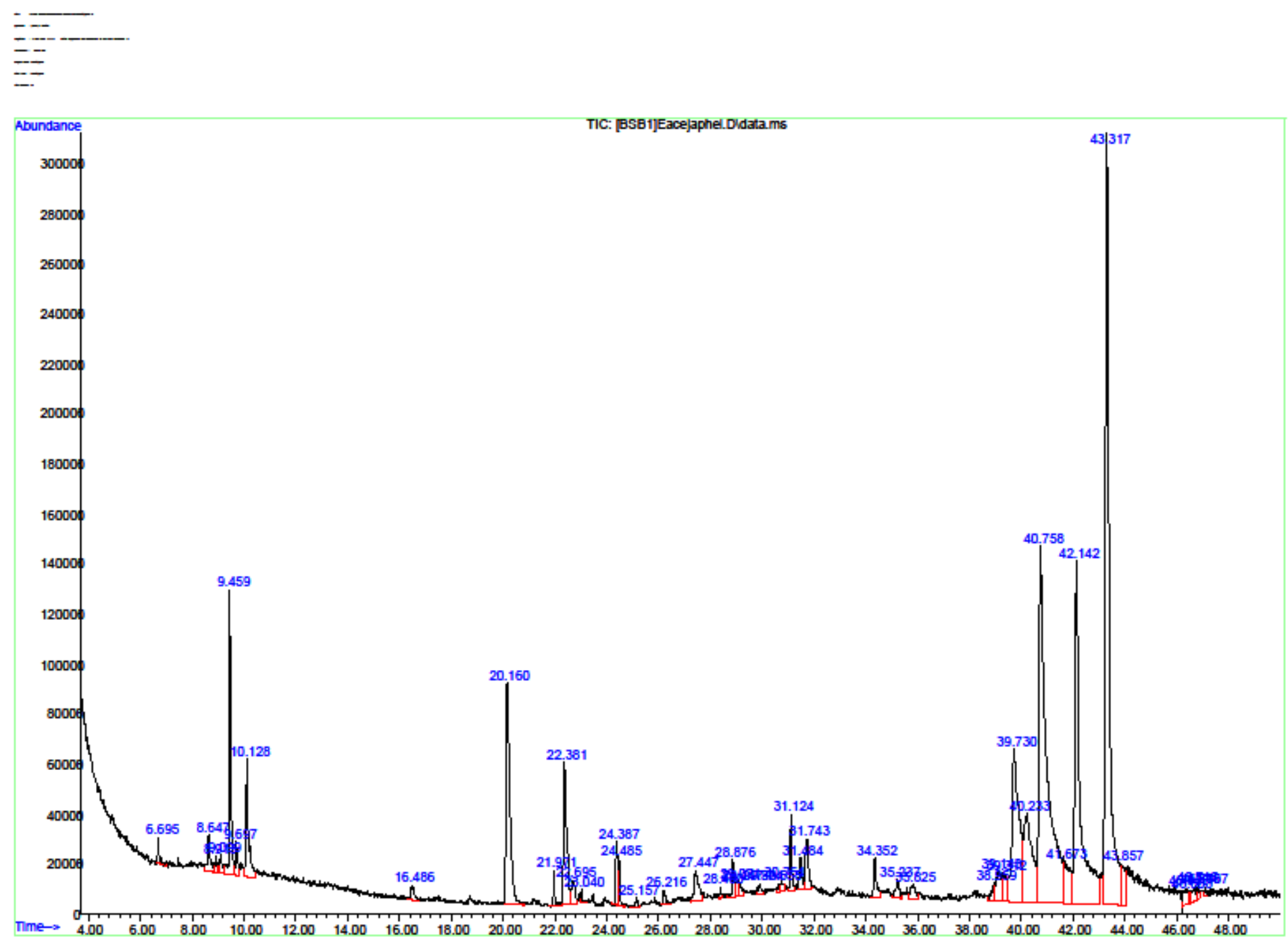

Figure 1

A chromatogram of the ethyl acetate leaf extract of 0 . basilicum 